\title{
A New Approach to the Method of Lyapunov Functionals and Its Applications
}

\author{
Yunguo Jin ${ }^{1}$ and Shouming Zhong ${ }^{1,2}$ \\ ${ }^{1}$ School of Mathematical Sciences, University of Electronic Science and Technology of China, Chengdu, Sichuan 611731, China \\ ${ }^{2}$ Key Laboratory for Neuroinformation of Ministry of Education, University of Electronic Science and Technology of China, \\ Chengdu, Sichuan 611731, China
}

Correspondence should be addressed to Yunguo Jin; yunguoj@163.com

Received 18 July 2013; Revised 2 November 2013; Accepted 3 November 2013

Academic Editor: Xinyu Song

Copyright (c) 2013 Y. Jin and S. Zhong. This is an open access article distributed under the Creative Commons Attribution License, which permits unrestricted use, distribution, and reproduction in any medium, provided the original work is properly cited.

\begin{abstract}
We show some results which can replace the graph theory used to construct global Lyapunov functions in some coupled systems of differential equations. We present an example of an epidemic model with stage structure and latency spreading in a heterogeneous host population and obtain a more general threshold for the extinction and persistence of a disease. Using some results obtained by mathematical induction and suitable Lyapunov functionals, we prove the global stability of the endemic equilibrium. For some coupled systems of differential equations, by a similar approach to the discussion of the epidemic model, the conditions of threshold property or global stability can be established without the assumption that the relative matrix is irreducible.
\end{abstract}

\section{Introduction}

Graph theory has developed into a substantial body of knowledge. A graph theoretic approach developed in $[1,2]$ is used to resolve a long-standing open problem on the uniqueness and global stability of the endemic equilibrium of a class of multigroup models in mathematical epidemiology. Using results from graph theory, a systematic approach developed in [3] allows one to construct global Lyapunov functions for large-scale coupled systems from building blocks of individual vertex systems. The graph-theoretical approach has been applied to various classes of coupled systems in engineering, ecology, and epidemiology (see, e.g., [1-13]). However, as in [14], while using the same Lyapunov function [3], sometimes graph theory can be replaced by positive operator theory. Furthermore, it seems that all authors use the graph theory under the assumption that the relative matrix is irreducible (see, e.g., [1-13]).

Motivated by the above discussion, in this paper, we show some results which can replace graph theory used to construct global Lyapunov functions in some coupled systems of differential equations (see, e.g., [1-13]) and a more general threshold without the assumption that the relative matrix is irreducible. For some coupled systems of differential equations (see, e.g., [1-13]), by a similar approach to the one discussed in this paper, the conditions of threshold property or global stability can be established without the assumption that the relative matrix is irreducible.

Various epidemics continue to pose a public health threat to humans. One of the most important subjects in this study of epidemic models (see, e.g., $[1-3,7-13]$ ) is to obtain a threshold that determines the persistence or extinction of a disease. In the real world, some epidemics, such as malaria, dengue, fever, gonorrhea, and bacterial infections, may have a different ability to transmit the infections in different ages. For example, measles and varicella always occur in juveniles, while it is reasonable to consider the disease transmission in adult population such as typhus and diphtheria. A heterogeneous host population can be divided into several homogeneous groups according to models of transmission, contact patterns, or geographic distributions. Since the time it takes from the moment of new infection to the moment of becoming infectious may differ from individual to individual, it is indeed a random variable. We present an example of an epidemic model with stage structure 
and latency spreading in a heterogeneous host population and show a general threshold to improved existing results.

Some results which can replace the graph theory used to construct global Lyapunov functions in some coupled systems of differential equations are shown in the next sections. In Section 3, a new approach to the method of Lyapunov functionals is applied to an epidemic model to obtain a more general threshold for the extinction and persistence of a disease.

\section{Some Results Obtained by Mathematical Induction}

In this section, we show some results which can replace graph theory used to construct global Lyapunov functions in some coupled systems of differential equations.

Assumption 1. There exist $v_{k}>0$, such that

$$
\sum_{j=1}^{n} \bar{\beta}_{k j} v_{k}-\sum_{j=1}^{n} \bar{\beta}_{j k} v_{j}=0
$$

where $\bar{\beta}_{k j} \geq 0, k, j=1,2, \ldots, n, n \geq 2$.

Theorem 2. Under Assumption 1, the following results hold:

$$
\sum_{k, j=1}^{n} v_{k} \bar{\beta}_{k j}\left[F_{k}\left(x_{k}\right)-F_{j}\left(x_{j}\right)\right]=0,
$$

where $F_{k}\left(x_{k}\right), k=1,2, \ldots, n$, are arbitrary functions. In particular, if $F_{k}\left(x_{k}\right)>0, k=1,2, \ldots, n$, then

$$
\sum_{k, j=1}^{n} v_{k} \bar{\beta}_{k j}\left[1-\frac{F_{k}\left(x_{k}\right)}{F_{j}\left(x_{j}\right)}\right] \leq 0
$$

Proof. Note that

$$
\begin{aligned}
& \sum_{k, j=1}^{n} v_{k} \bar{\beta}_{k j}\left[F_{k}\left(x_{k}\right)-F_{j}\left(x_{j}\right)\right] \\
& \quad=\sum_{n \geq k \geq j \geq 1}\left(v_{k} \bar{\beta}_{k j}-v_{j} \bar{\beta}_{j k}\right)\left[F_{k}\left(x_{k}\right)-F_{j}\left(x_{j}\right)\right] \\
& =: Z_{n} .
\end{aligned}
$$

Let

$$
\begin{array}{r}
\Delta_{L}=\sum_{L \geq k \geq j \geq 2}\left(v_{k} \bar{\beta}_{k j}-v_{j} \bar{\beta}_{j k}\right)\left[F_{k}\left(x_{k}\right)-F_{j}\left(x_{j}\right)\right], \\
L=2,3, \ldots, n .
\end{array}
$$

According to Assumption 1, it is easy to see that we only need to prove the following result:

$$
\begin{gathered}
Z_{n}=\sum_{L=2}^{q+1}\left(v_{L} \bar{\beta}_{L 1}-v_{1} \bar{\beta}_{1 L}+v_{L} \bar{\beta}_{L n}-v_{n} \bar{\beta}_{n L}+v_{L} \bar{\beta}_{L, n-1}\right. \\
\left.-v_{n-1} \bar{\beta}_{n-1, L}+\cdots+v_{L} \bar{\beta}_{L, q+2}-v_{q+2} \bar{\beta}_{q+2, L}\right) \\
\times\left[F_{L}\left(x_{L}\right)-F_{1}\left(x_{1}\right)\right]+\Delta_{q+1}=: A_{q+1}, \\
q=1,2, \ldots, n-2 .
\end{gathered}
$$

In fact, if the above result holds, then we have

$$
Z_{n}=\sum_{L=1}^{n}\left(v_{2} \bar{\beta}_{2 L}-v_{L} \bar{\beta}_{L 2}\right)\left[F_{2}\left(x_{2}\right)-F_{1}\left(x_{1}\right)\right]+\Delta_{2}
$$

Using Assumption 1, we have $Z_{n}=\Delta_{2}=0$. First, we show that $Z_{n}=A_{n-1}$. We can rewrite $Z_{n}$ as

$$
\begin{aligned}
Z_{n}=\sum_{L=2}^{n}\left\{\left(v_{n} \bar{\beta}_{n L}-v_{L} \bar{\beta}_{L n}\right)\left[F_{n}\left(x_{n}\right)-F_{L}\left(x_{L}\right)\right]\right. \\
\left.+\left(v_{L} \bar{\beta}_{L 1}-v_{1} \bar{\beta}_{1 L}\right)\left[F_{L}\left(x_{L}\right)-F_{1}\left(x_{1}\right)\right]\right\}+\Delta_{n-1} .
\end{aligned}
$$

Then, using the fact that $a b+c d=a(b+d)+d(c-a)$, where $a, b, c$, and $d$ are arbitrary numbers, we may obtain

$$
\begin{aligned}
Z_{n}=\sum_{L=2}^{n}\{ & \left(v_{n} \bar{\beta}_{n L}-v_{L} \bar{\beta}_{L n}\right)\left[F_{n}\left(x_{n}\right)-F_{1}\left(x_{1}\right)\right] \\
& +\left(v_{L} \bar{\beta}_{L 1}-v_{1} \bar{\beta}_{1 L}+v_{L} \bar{\beta}_{L n}-v_{n} \bar{\beta}_{n L}\right) \\
& \left.\times\left[F_{L}\left(x_{L}\right)-F_{1}\left(x_{1}\right)\right]\right\}+\Delta_{n-1} \\
= & \sum_{L=2}^{n}\left(v_{n} \bar{\beta}_{n L}-v_{L} \bar{\beta}_{L n}\right)\left[F_{n}\left(x_{n}\right)-F_{1}\left(x_{1}\right)\right] \\
& +\left(v_{n} \bar{\beta}_{n 1}-v_{1} \bar{\beta}_{1 n}\right)\left[F_{n}\left(x_{n}\right)-F_{1}\left(x_{1}\right)\right] \\
& +\sum_{L=2}^{n-1}\left(v_{L} \bar{\beta}_{L 1}-v_{1} \bar{\beta}_{1 L}+v_{L} \bar{\beta}_{L n}-v_{n} \bar{\beta}_{n L}\right) \\
= & \times\left[F_{L}\left(x_{L}\right)-F_{1}\left(x_{1}\right)\right]+\Delta_{n-1} \\
& \sum_{L=1}^{n}\left(v_{n} \bar{\beta}_{n L}-v_{L} \bar{\beta}_{L n}\right)\left[F_{n}\left(x_{n}\right)-F_{1}\left(x_{1}\right)\right]+\Delta_{n-1} \\
& +\sum_{L=2}^{n-1}\left(v_{L} \bar{\beta}_{L 1}-v_{1} \bar{\beta}_{1 L}+v_{L} \bar{\beta}_{L n}-v_{n} \bar{\beta}_{n L}\right) \\
& \times\left[F_{L}\left(x_{L}\right)-F_{1}\left(x_{1}\right)\right] .
\end{aligned}
$$


Using Assumption 1, we have $Z_{n}=A_{n-1}$. Next, we show that $A_{q+1}=A_{q}, q=1,2, \ldots, n-2$. We can rewrite $A_{q+1}$ as

$$
\begin{aligned}
A_{q+1}=\sum_{L=2}^{q+1}\left(v_{L} \bar{\beta}_{L 1}-v_{1} \bar{\beta}_{1 L}+v_{L} \bar{\beta}_{L n}-v_{n} \bar{\beta}_{n L}+v_{L} \bar{\beta}_{L, n-1}\right. \\
\left.\quad-v_{n-1} \bar{\beta}_{n-1, L}+\cdots+v_{L} \bar{\beta}_{L, q+2}-v_{q+2} \bar{\beta}_{q+2, L}\right) \\
\quad \times\left[F_{L}\left(x_{L}\right)-F_{1}\left(x_{1}\right)\right]+\Delta_{q} \\
+\sum_{j=2}^{q+1}\left(v_{q+1} \bar{\beta}_{q+1, j}-v_{j} \bar{\beta}_{j, q+1}\right)\left[F_{q+1}\left(x_{q+1}\right)-F_{j}\left(x_{j}\right)\right] .
\end{aligned}
$$

By a similar argument as for the discussion

$$
\begin{aligned}
& \sum_{L=2}^{n}\{\left(v_{n} \bar{\beta}_{n L}-v_{L} \bar{\beta}_{L n}\right)\left[F_{n}\left(x_{n}\right)-F_{L}\left(x_{L}\right)\right] \\
&+\left.\left(v_{L} \bar{\beta}_{L 1}-v_{1} \bar{\beta}_{1 L}\right)\left[F_{L}\left(x_{L}\right)-F_{1}\left(x_{1}\right)\right]\right\} \\
&= \sum_{L=1}^{n}\left(v_{n} \bar{\beta}_{n L}-v_{L} \bar{\beta}_{L n}\right)\left[F_{n}\left(x_{n}\right)-F_{1}\left(x_{1}\right)\right] \\
&+\sum_{L=2}^{n-1}\left(v_{L} \bar{\beta}_{L 1}-v_{1} \bar{\beta}_{1 L}+v_{L} \bar{\beta}_{L n}-v_{n} \bar{\beta}_{n L}\right) \\
& \quad \times\left[F_{L}\left(x_{L}\right)-F_{1}\left(x_{1}\right)\right],
\end{aligned}
$$

in the proof of $Z_{n}=A_{n-1}$, we can obtain

$$
\begin{aligned}
\sum_{L=2}^{q+1}\left(v_{L} \bar{\beta}_{L 1}-v_{1} \bar{\beta}_{1 L}\right)\left[F_{L}\left(x_{L}\right)-F_{1}\left(x_{1}\right)\right] \\
+\sum_{j=2}^{q+1}\left(v_{q+1} \bar{\beta}_{q+1, j}-v_{j} \bar{\beta}_{j, q+1}\right)\left[F_{q+1}\left(x_{q+1}\right)-F_{j}\left(x_{j}\right)\right] \\
=\sum_{L=1}^{q+1}\left(v_{q+1} \bar{\beta}_{q+1, L}-v_{L} \bar{\beta}_{L, q+1}\right)\left[F_{q+1}\left(x_{q+1}\right)-F_{1}\left(x_{1}\right)\right] \\
+\sum_{L=2}^{q}\left(v_{L} \bar{\beta}_{L 1}-v_{1} \bar{\beta}_{1 L}+v_{L} \bar{\beta}_{L, q+1}-v_{q+1} \bar{\beta}_{q+1, L}\right) \\
\quad \times\left[F_{L}\left(x_{L}\right)-F_{1}\left(x_{1}\right)\right] .
\end{aligned}
$$

Substituting (12) into (10), we have

$$
\begin{aligned}
A_{q+1}=\sum_{L=2}^{q+1}\left(v_{L} \bar{\beta}_{L n}-v_{n} \bar{\beta}_{n L}+v_{L} \bar{\beta}_{L, n-1}-v_{n-1} \bar{\beta}_{n-1, L}\right. \\
\left.\quad+\cdots+v_{L} \bar{\beta}_{L, q+2}-v_{q+2} \bar{\beta}_{q+2, L}\right) \\
\quad \times\left[F_{L}\left(x_{L}\right)-F_{1}\left(x_{1}\right)\right]+\Delta_{q} \\
+\sum_{L=1}^{q+1}\left(v_{q+1} \bar{\beta}_{q+1, L}-v_{L} \bar{\beta}_{L, q+1}\right)\left[F_{q+1}\left(x_{q+1}\right)-F_{1}\left(x_{1}\right)\right]
\end{aligned}
$$

$$
\begin{aligned}
& +\sum_{L=2}^{q}\left(v_{L} \bar{\beta}_{L 1}-v_{1} \bar{\beta}_{1 L}+v_{L} \bar{\beta}_{L, q+1}-v_{q+1} \bar{\beta}_{q+1, L}\right) \\
& \quad \times\left[F_{L}\left(x_{L}\right)-F_{1}\left(x_{1}\right)\right] \\
& =\sum_{L=1}^{n}\left(v_{q+1} \bar{\beta}_{q+1, L}-v_{L} \bar{\beta}_{L, q+1}\right)\left[F_{q+1}\left(x_{q+1}\right)-F_{1}\left(x_{1}\right)\right] \\
& +A_{q} .
\end{aligned}
$$

By Assumption 1, we can get $A_{q+1}=A_{q}$. Using 1 $\left(F_{k}\left(x_{k}\right) / F_{j}\left(x_{j}\right)\right)+\ln \left(F_{k}\left(x_{k}\right) / F_{j}\left(x_{j}\right)\right) \leq 0$ and the result above, we can obtain that $\sum_{k, j=1}^{n} v_{k} \bar{\beta}_{k j}\left(1-\left(F_{k}\left(x_{k}\right) / F_{j}\left(x_{j}\right)\right)\right) \leq 0$. This completes the proof.

Remark 3. Some results in [1-13] from graph theory can be obtained by Theorem 2 . For example, $\sum_{k, j=1}^{n} v_{k} \bar{\beta}_{k j} \ln \left(\left(E_{k} E_{j}^{*}\right) /\left(E_{k}^{*} E_{j}\right)\right)=0$ in [11] and $\sum_{k, j=1}^{n} v_{k} \bar{\beta}_{k j}\left[n+2-\left(S_{k}^{*} / S_{k}\right)-\left(\left(S_{k} I_{j} y_{k, 1}^{*}\right) /\left(S_{k}^{*} I_{j}^{*} y_{k, 1}\right)\right)-\right.$ $\left.\sum_{i=2}^{n}\left(\left(y_{k, i-1} y_{k, i}^{*}\right) /\left(y_{k, i-1}^{*} y_{k, i}\right)\right)-\left(\left(y_{k, n} I_{k}^{*}\right) /\left(y_{k, n}^{*} I_{k}\right)\right) \leq 0\right]$ in [10].

The following result is one result of Kirchhoff's Three Theorem (a result in graph theory). In the following, using mathematical induction, we show the result.

Theorem 4. If $\left(\bar{\beta}_{k j}\right)_{n \times n}, n \geq 2$, is irreducible, then Assumption 1 holds.

Proof. Obviously, the result holds for $n=2$. We assume $n \geq 3$ and can rewrite (1) as

$$
\left[\begin{array}{cccc}
\sum_{l \neq 1} \bar{\beta}_{1 l} & -\bar{\beta}_{21} & \cdots & -\bar{\beta}_{n 1} \\
-\bar{\beta}_{12} & \sum_{l \neq 2} \bar{\beta}_{2 l} & \cdots & -\bar{\beta}_{n 2} \\
\vdots & \vdots & \ddots & \vdots \\
-\bar{\beta}_{1 n} & -\overline{\bar{\beta}}_{2 n} & \cdots & \sum_{l \neq n} \bar{\beta}_{n l}
\end{array}\right]\left(\begin{array}{c}
v_{1} \\
v_{2} \\
\vdots \\
v_{n}
\end{array}\right)=\left(\begin{array}{c}
0 \\
0 \\
\vdots \\
0
\end{array}\right) .
$$

Note that (14) is equivalent to the following system:

$$
\begin{gathered}
{\left[\begin{array}{cccc}
\sum_{l \neq 1} \bar{\beta}_{1 l} & -\bar{\beta}_{21} & \cdots & -\bar{\beta}_{n 1} \\
0 & \sum_{l \neq 2} \bar{\beta}_{2 l}-\bar{\beta}_{21} \frac{\bar{\beta}_{12}}{\sum_{l \neq 1} \bar{\beta}_{1 l}} & \cdots & -\bar{\beta}_{n 2}-\bar{\beta}_{n 1} \frac{\bar{\beta}_{12}}{\sum_{l \neq 1} \bar{\beta}_{1 l}} \\
\vdots & \vdots & \ddots & \vdots \\
0 & -\bar{\beta}_{2 n}-\bar{\beta}_{21} \frac{\bar{\beta}_{1 n}}{\sum_{l \neq 1} \bar{\beta}_{1 l}} & \cdots & \sum_{l \neq n} \bar{\beta}_{n l}-\bar{\beta}_{n 1} \frac{\bar{\beta}_{1 n}}{\sum_{l \neq 1} \bar{\beta}_{1 l}}
\end{array}\right]} \\
\quad \times\left(\begin{array}{c}
v_{1} \\
v_{2} \\
\vdots \\
v_{n}
\end{array}\right)=\left(\begin{array}{c}
0 \\
0 \\
\vdots \\
0
\end{array}\right) .
\end{gathered}
$$


Consider the following system:

$$
\begin{gathered}
{\left[\begin{array}{ccccc}
\sum_{l \neq 2} \bar{\beta}_{2 l}-\bar{\beta}_{21} \frac{\bar{\beta}_{12}}{\sum_{l \neq 1} \bar{\beta}_{1 l}} & -\bar{\beta}_{32}-\bar{\beta}_{31} \frac{\bar{\beta}_{12}}{\sum_{l \neq 1} \bar{\beta}_{1 l}} & \cdots & -\bar{\beta}_{n 2}-\bar{\beta}_{n 1} \frac{\bar{\beta}_{12}}{\sum_{l \neq \neq} \bar{\beta}_{1 l}} \\
-\bar{\beta}_{23}-\bar{\beta}_{21} \frac{\bar{\beta}_{13}}{\sum_{l \neq 1} \bar{\beta}_{1 l}} & \sum_{l \neq 3} \bar{\beta}_{3 l}-\bar{\beta}_{31} \frac{\bar{\beta}_{13}}{\sum_{l \neq 1} \bar{\beta}_{1 l}} & \cdots & -\bar{\beta}_{n 3}-\bar{\beta}_{n 1} \frac{\bar{\beta}_{13}}{\sum_{l \neq 1} \bar{\beta}_{1 l}} \\
\vdots & \vdots & \ddots & \vdots \\
-\bar{\beta}_{2 n}-\bar{\beta}_{21} \frac{\bar{\beta}_{1 n}}{\sum_{l \neq 1} \bar{\beta}_{1 l}} & -\bar{\beta}_{3 n}-\bar{\beta}_{31} \frac{\bar{\beta}_{1 n}}{\sum_{l \neq 1} \bar{\beta}_{1 l}} & \cdots & \sum_{l \neq n} \bar{\beta}_{n l}-\bar{\beta}_{n 1} \frac{\bar{\beta}_{1 n}}{\sum_{l \neq 1} \bar{\beta}_{1 l}}
\end{array}\right]} \\
\quad \times\left(\begin{array}{c}
v_{2} \\
\vdots \\
v_{n}
\end{array}\right)=\left(\begin{array}{c}
0 \\
\vdots \\
0
\end{array}\right) .
\end{gathered}
$$

$$
\begin{aligned}
& {\left[\begin{array}{cccc}
-a_{11} & -a_{21} & \cdots & -a_{n-1,1} \\
-a_{12} & -a_{22} & \cdots & -a_{n-1,2} \\
\vdots & \vdots & \ddots & \vdots \\
-a_{1, n-1} & -a_{2, n-1} & \cdots & -a_{n-1, n-1}
\end{array}\right]} \\
& =\left[\begin{array}{cccccc}
\sum_{l \neq 2} \bar{\beta}_{2 l}-\bar{\beta}_{21} \frac{\bar{\beta}_{12}}{\sum_{l \neq 1} \bar{\beta}_{1 l}} & -\bar{\beta}_{32}-\bar{\beta}_{31} \frac{\bar{\beta}_{12}}{\sum_{l \neq 1} \bar{\beta}_{1 l}} & \cdots & -\bar{\beta}_{n 2}-\bar{\beta}_{n 1} \frac{\bar{\beta}_{12}}{\sum_{l \neq 1} \bar{\beta}_{1 l}} \\
-\bar{\beta}_{23}-\bar{\beta}_{21} \frac{\bar{\beta}_{13}}{\sum_{l \neq 1} \bar{\beta}_{1 l}} & \sum_{l \neq 3} \bar{\beta}_{3 l}-\bar{\beta}_{31} \frac{\bar{\beta}_{13}}{\sum_{l \neq 1} \bar{\beta}_{1 l}} & \cdots & -\bar{\beta}_{n 3}-\bar{\beta}_{n 1} \frac{\bar{\beta}_{13}}{\sum_{l \neq 1} \bar{\beta}_{1 l}} \\
\vdots & \bar{\beta}_{1 n} & -\bar{\beta}_{3 n}-\bar{\beta}_{31} \frac{\bar{\beta}_{1 n}}{\sum_{l \neq 1} \bar{\beta}_{1 l}} & \cdots & \sum_{l \neq n} \bar{\beta}_{n l}-\bar{\beta}_{n 1} \frac{\bar{\beta}_{1 n}}{\sum_{l \neq 1} \bar{\beta}_{1 l}}
\end{array}\right] .
\end{aligned}
$$

We can rewrite (16) as

$$
\left[\begin{array}{cccc}
\sum_{l \neq 1} a_{1 l} & -a_{21} & \cdots & -a_{n-1,1} \\
-a_{12} & \sum_{l \neq 2} a_{2 l} & \cdots & -a_{n-1,2} \\
\vdots & \vdots & \ddots & \vdots \\
-a_{1, n-1} & -a_{2, n-1} & \cdots & \sum_{l \neq n-1} a_{n-1, l}
\end{array}\right]\left(\begin{array}{c}
v_{2} \\
v_{3} \\
\vdots \\
v_{n}
\end{array}\right)=\left(\begin{array}{c}
0 \\
0 \\
\vdots \\
0
\end{array}\right)
$$

From (17), it is easy to see that $\left(a_{k j}\right)_{(n-1) \times(n-1)}, n \geq 3$, is also irreducible. Note that $\sum_{l \neq 1} \bar{\beta}_{1 l} \neq 0$. According to the discussion above, we can deduce that if the result (if $\left(\bar{\beta}_{k j}\right)_{n \times n}$, $n \geq 2$, is irreducible, then Assumption 1 holds) holds for
$n=L, L \geq 2$, then it holds also for $n=L+1$. The proof is complete.

\section{An Example of an Epidemic Model with Stage Structure and Latency Spreading in a Heterogeneous Host Population}

In this section, we present an epidemic model with stage structure and latency spreading in a heterogeneous host population and obtain a more general threshold for the extinction and persistence of a disease. Using the results obtained by mathematical induction and suitable Lyapunov functionals, we prove the global stability of the endemic equilibrium. For some coupled systems of differential equations, by a 
similar approach to the discussion of the epidemic model, the conditions of threshold property or global stability can be established without the assumption that the relative matrix is irreducible.

We formulate an epidemic model with latency spreading in a heterogeneous host population. Let $S_{k}^{(1)}, S_{k}^{(2)}, I_{k}$, and $R_{k}$ denote the immature susceptible, mature susceptible, infectious, and recovered population in the $k$ th group, respectively. The disease incidence in the $k$ th group can be calculated as

$$
\sum_{i=1}^{2} \sum_{j=1}^{n} \beta_{k j}^{(i)} S_{k}^{(i)} I_{j}
$$

where the sum takes into account cross-infections from all groups and $\beta_{k j}^{(i)}$ is the transmission coefficient between compartments $S_{k}^{(i)}$ and $I_{j}$. Let $d_{k}^{(1)}$ and $d_{k}^{(2)}$ represent death rates of $S_{k}^{(1)}$ and $S_{k}^{(2)}$ populations, respectively. Let $\tau$ be the latent period of the population. $e^{-d_{k}^{(i)} \tau} \beta_{k j}^{(i)} S_{k}^{(i)}(t-\tau) I_{j}(t-\tau)$ represents the individuals surviving in the latent period $\tau$ and becoming infective at time $t$. Let $p_{k}^{(i)}(\tau):[0, h] \rightarrow \mathbb{R}_{+}$be integrable function with $\int_{0}^{h} p_{k}^{(i)}(\tau) d \tau=1$. We assume that $\tau$ is distributed according to $p_{k}^{(i)}(\tau)$ over the interval $[0, h]$, where $h$ is the upper bound of the latent period. Then, we obtain the following model for a disease with latency:

$$
\begin{gathered}
\dot{S}_{k}^{(1)}=b_{k}-d_{k}^{(1)} S_{k}^{(1)}-\sum_{j=1}^{n} \beta_{k j}^{(1)} S_{k}^{(1)} I_{j}-a_{k} S_{k}^{(1)}, \\
\dot{S}_{k}^{(2)}=a_{k} S_{k}^{(1)}-\sum_{j=1}^{n} \beta_{k j}^{(2)} S_{k}^{(2)} I_{j}-d_{k}^{(2)} S_{k}^{(2)}, \\
\dot{I}_{k}=\sum_{i=1}^{2} \sum_{j=1}^{n} \int_{0}^{h} p_{k}^{(i)}(\tau) e^{-d_{k}^{(i)} \tau} \beta_{k j}^{(i)} S_{k}^{(i)}(t-\tau) I_{j}(t-\tau) d \tau \\
-\left(d_{k}+\mu_{k}+\gamma_{k}\right) I_{k}, \\
\dot{R}_{k}=\gamma_{k} I_{k}-d_{k} R_{k}, \quad k=1,2, \ldots, n,
\end{gathered}
$$

where $b_{k}^{(1)}$ denotes influx of individuals into the immature susceptible class in the $k$ th group. $a_{k}$ is the conversion rate from immature individual to mature individual in group $k$. $d_{k}, \mu_{k}$, and $\gamma_{k}$ are the natural death rate, the disease-related death rate, and the recovery rate in the $k$ th group, respectively. All parameter values are assumed to be nonnegative and $b_{k}$, $h, a_{k}, d_{k}^{(i)}, d_{k}>0$.

Let $f_{k}^{(i)}(\tau)=p_{k}^{(i)}(\tau) e^{-d_{k}^{(i)} \tau}, c_{k}^{(i)}=\int_{0}^{h} p_{k}^{(i)}(\tau) e^{-d_{k}^{(i)} \tau} d \tau$, $\varphi_{k}\left(S_{k}^{(1)}\right)=b_{k}-d_{k}^{(1)} S_{k}^{(1)}$, and $m_{k}=d_{k}+\mu_{k}+\gamma_{k}$. Since the variables $R_{k}$ do not appear in the remaining three equations of (20), we can consider the following reduced system:

$$
\begin{array}{r}
\dot{S}_{k}^{(1)}=\varphi_{k}\left(S_{k}^{(1)}\right)-\sum_{j=1}^{n} \beta_{k j}^{(1)} S_{k}^{(1)} I_{j}-a_{k} S_{k}^{(1)}, \\
\dot{S}_{k}^{(2)}=a_{k} S_{k}^{(1)}-\sum_{j=1}^{n} \beta_{k j}^{(2)} S_{k}^{(2)} I_{j}-d_{k}^{(2)} S_{k}^{(2)}, \\
\dot{I}_{k}=\sum_{i=1}^{2} \sum_{j=1}^{n} \int_{0}^{h} f_{k}^{(i)}(\tau) \beta_{k j}^{(i)} S_{k}^{(i)}(t-\tau) I_{j}(t-\tau) d \tau-m_{k} I_{k}, \\
k=1,2, \ldots, n .
\end{array}
$$

The initial conditions for system (21) take the form

$$
\begin{aligned}
& S_{k}^{(1)}(\theta)=\phi_{k}^{(1)}(\theta) \geq 0, \quad S_{k}^{(2)}(\theta)=\phi_{k}^{(2)}(\theta) \geq 0, \\
& I_{k}(\theta)=\phi_{k}(\theta) \geq 0, \quad \phi_{k}^{(i)}(0)>0, \quad \phi_{k}(0)>0, \\
& i=1,2, \quad k=1,2, \ldots, n, \quad \theta \in[-h, 0],
\end{aligned}
$$

where $\left(\phi_{1}^{(1)}(\theta), \phi_{1}^{(2)}(\theta), \ldots, \phi_{n}(\theta)\right) \in C\left([-h, 0], \mathbb{R}_{+}^{3 n}\right)$, the Banach space of continuous functions mapping the interval $[-h, 0]$ into $\mathbb{R}_{+}^{3 n}$.

We see that system (21) exits a disease-free equilibrium $P_{0}=\left(S_{10}^{(1)}, S_{10}^{(2)}, \ldots, S_{n 0}^{(1)}, S_{n 0}^{(2)}, 0,0, \ldots, 0\right)$, where

$$
\varphi_{k}\left(S_{k 0}^{(1)}\right)=d_{k}^{(2)} S_{k 0}^{(2)}, \quad a_{k} S_{k 0}^{(1)}=d_{k}^{(2)} S_{k 0}^{(2)}, \quad k=1,2, \ldots, n .
$$

Let

$$
A_{k j}=\frac{\sum_{i=1}^{2} c_{k}^{(i)} \beta_{k j}^{(i)} S_{k 0}^{(i)}}{m_{k}}, \quad k, j=1,2, \ldots, n
$$

and $\mathbf{Q}=\left(A_{k j}\right)_{n \times n}$. We assume that

(H1) there exist $\left(w_{1}, w_{2}, \ldots, w_{n}\right)>0\left(w_{k}>0, k=\right.$ $1,2, \ldots, n)$, such that

$$
\left(w_{1}, w_{2}, \ldots, w_{n}\right) \mathbf{Q}<\left(w_{1}, w_{2}, \ldots, w_{n}\right) ;
$$

(H2) there exist $\left(w_{1}, w_{2}, \ldots, w_{n}\right)>0$, such that

$$
\left(w_{1}, w_{2}, \ldots, w_{n}\right) \mathbf{Q}=\left(w_{1}, w_{2}, \ldots, w_{n}\right) ;
$$

(H3) there exist $\left(w_{1}, w_{2}, \ldots, w_{n}\right)>0$, such that

$$
\left(w_{1}, w_{2}, \ldots, w_{n}\right) \mathbf{Q}>\left(w_{1}, w_{2}, \ldots, w_{n}\right) .
$$

Let

(i) $R_{0}<1$ if and only if (H1) holds;

(ii) $R_{0}=1$ if and only if (H2) holds;

(iii) $R_{0}>1$ if and only if (H3) holds. 
Remark 5. By an approach as the one in $[1-3,7-13]$, we define $r_{0}$. Let $r_{0}=\rho(\mathbf{Q})$, where $\rho$ denotes the spectral radius. If a matrix is irreducible, then, for the eigenvalue of maximum, the associated eigenvector is positive. Note that the authors in $[1-3,7-13]$ discussed some coupled systems of differential equations under a definition with an approach as the one of definition of $r_{0}$ and the assumption that the relevant matrix is irreducible. In fact, if $r_{0}<1$ and the relevant matrix is irreducible, then $R_{0}<1$; if $r_{0}=1$ and the relevant matrix is irreducible, then $R_{0}=1$; if $r_{0}>1$ and the relevant matrix is irreducible, then $R_{0}>1$. However, the reverse is not true. For example,

$$
\left(A_{k j}\right)_{2 \times 2}=\left[\begin{array}{ll}
1 & 0 \\
1 & 2
\end{array}\right] .
$$

Furthermore, let

$$
\left(A_{k j}\right)_{4 \times 4}=\left[\begin{array}{llll}
1 & 1 & 0 & 0 \\
1 & 1 & 0 & 0 \\
0 & 0 & 1 & 1 \\
0 & 0 & 1 & 1
\end{array}\right] .
$$

Obviously, $R_{0}>1$ holds also for arbitrary $\left(w_{1}, w_{2}, w_{3}, w_{4}\right)>$ 0. $\left(A_{k j}\right)_{4 \times 4}$ conforms to the conditions of Theorem 8 but is not in accord with the conditions of Corollary 10. The definition of $r_{0}$ and the assumption that the relative matrix is irreducible in the results of Corollaries 9 and 10 are analogous with relative definition and assumption in [1-3, 7-13].

The equilibria of (21) are the same as those of the associated ODE system:

$$
\begin{gathered}
\dot{S}_{k}^{(1)}=\varphi_{k}\left(S_{k}^{(1)}\right)-S_{k}^{(1)} \sum_{j=1}^{n} \beta_{k j}^{(1)} I_{j}-a_{k} S_{k}^{(1)}, \\
\dot{S}_{k}^{(2)}=a_{k} S_{k}^{(1)}-S_{k}^{(2)} \sum_{j=1}^{n} \beta_{k j}^{(2)} I_{j}-d_{k}^{(2)} S_{k}^{(2)}, \\
\dot{I}_{k}=\sum_{i=1}^{2} c_{k}^{(i)} S_{k}^{(i)} \sum_{j=1}^{n} \beta_{k j}^{(i)} I_{j}-m_{k} I_{k}, \quad k=1,2, \ldots, n .
\end{gathered}
$$

Let

$$
\begin{array}{r}
N_{k}=S_{k}^{(1)}+S_{k}^{(2)}+I_{k}, \quad \underline{d}_{k}=\min \left\{d_{k}^{(1)}, d_{k}^{(2)}, d_{k}\right\}, \\
k=1,2, \ldots, n .
\end{array}
$$

From (30) and $c_{k}^{(i)} \leq 1$, we have

$$
\dot{N}_{k} \leq \varphi_{k}\left(S_{k}^{(1)}\right)+d_{k}^{(1)} S_{k}^{(1)}-\underline{d}_{k} N_{k} .
$$

We derive from (32) that the region

$$
\begin{aligned}
\Gamma=\{ & \left(S_{1}^{(1)}, S_{1}^{(2)}, \ldots, S_{n}^{(1)}, S_{n}^{(2)}, I_{1}, \ldots, I_{n}\right) \\
& \in \mathbb{R}_{+}^{3 n}: S_{k}^{(1)} \leq S_{k 0}^{(1)}, S_{k}^{(2)} \leq S_{k 0}^{(2)}, S_{k}^{(1)}+S_{k}^{(2)}+I_{k} \\
& \left.\leq \frac{\varphi_{k}(0)+d_{k}^{(1)} S_{k 0}^{(1)}}{d_{k}}, k=1,2, \ldots, n\right\}
\end{aligned}
$$

is a forward invariant compact absorbing set with respect to (30). Let $\Gamma^{\circ}$ denote the interior of $\Gamma$.

Note that $\Gamma$ is positively invariant with respect to (21). In fact, let

$$
\begin{array}{r}
\dot{E}_{k}=\sum_{i=1}^{2} \sum_{j=1}^{n} \beta_{k j}^{(i)} S_{k}^{(i)} I_{j} \\
-\sum_{i=1}^{2} \sum_{j=1}^{n} \int_{0}^{h} f_{k}^{(i)}(\tau) \beta_{k j}^{(i)} S_{k}^{(i)}(t-\tau) I_{j}(t-\tau) d \tau-d_{k} E_{k}, \\
k=1,2, \ldots, n .
\end{array}
$$

Then, we have

$$
\dot{N}_{k}+\dot{E}_{k} \leq \varphi_{k}\left(S_{k}^{(1)}\right)+d_{k}^{(1)} S_{k}^{(1)}-\underline{d}_{k}\left(N_{k}+E_{k}\right) .
$$

Furthermore, using the fact that $N_{k} \leq N_{k}+E_{k}$, we may obtain that $\Gamma$ is positively invariant with respect to (21).

Lemma 6. If $R_{0}>1$, then $P_{0}$ of system (30) is unstable in $\Gamma$ and there exists a positive equilibrium $P^{*}$ in $\Gamma^{\circ}$.

Proof. Let $\mathbf{I}=\left(I_{1}, I_{2}, \ldots, I_{n}\right)$ and $M=\sum_{k=1}^{n}\left(\left(\omega_{k} I_{k}\right) /\left(m_{k}\right)\right)$. Thus

$$
\begin{aligned}
\dot{M} & =\sum_{k=1}^{n} \omega_{k}\left[\frac{\sum_{i=1}^{2} c_{k}^{(i)} \sum_{j=1}^{n} \beta_{k j}^{(i)} S_{k}^{(i)} I_{j}}{m_{k}}-I_{k}\right] \\
& \leq\left(\omega_{1}, \omega_{2}, \ldots, \omega_{n}\right)\left(\mathbf{Q} \mathbf{I}^{T}-\mathbf{I}^{T}\right) .
\end{aligned}
$$

If $R_{0}>1$, by continuity, we obtain $\dot{M}>0$, in a neighborhood of $P_{0}$ in $\Gamma^{\circ}$. This implies that $P_{0}$ is unstable. Using the uniform persistence result from [15] and by a similar argument to that in the proof in [1], we can show that if $R_{0}>1$, the instability of $P_{0}$ implies the uniform persistence of system (30). This, together with the uniform boundedness of solutions of (30) in $\Gamma^{\circ}$, implies (30) has at least a positive equilibrium $P^{*}$ in $\Gamma^{\circ}$. The proof is completed.

Let

$$
P^{*}=\left(S_{1}^{(1) *}, S_{1}^{(2) *}, \ldots, S_{n}^{(1) *}, S_{n}^{(2) *}, I_{1}^{*}, I_{2}^{*}, \ldots, I_{n}^{*}\right) ;
$$

then the components of $P^{*}$ satisfy

$$
\begin{gathered}
\varphi_{k}\left(S_{k}^{(1) *}\right)=\sum_{i=1}^{2} S_{k}^{(i) *} \sum_{j=1}^{n} \beta_{k j}^{(i)} I_{j}^{*}+d_{k}^{(2)} S_{k}^{(2) *}, \\
\sum_{i=1}^{2} c_{k}^{(i)} S_{k}^{(i) *} \sum_{j=1}^{n} \beta_{k j}^{(i)} I_{j}^{*}=m_{k} I_{k}^{*}, \\
a_{k} S_{k}^{(1) *}=d_{k}^{(2)} S_{k}^{(2) *}+S_{k}^{(2) *} \sum_{j=1}^{n} \beta_{k j}^{(2)} I_{j}^{*}, \quad k=1,2, \ldots, n .
\end{gathered}
$$

Next, we will study the global stability of equilibria of system (21). 
Theorem 7. If $c_{k}^{(1)} \geq c_{k}^{(2)}, k=1,2, \ldots, n$, and $R_{0} \leq 1$, then $P_{0}$ of system (21) is globally asymptotically stable in $\Gamma$.

Proof. Let $\mathbf{S}^{\mathbf{0}}=\left(S_{10}^{(1)}, S_{10}^{(2)}, \ldots, S_{n 0}^{(1)}, S_{n 0}^{(2)}\right)$. Consider a Lyapunov functional $L_{1}+L_{2}$, where

$$
\begin{gathered}
L_{1}=\sum_{k=1}^{n} \frac{\omega_{k}}{m_{k}}\left[\sum_{i=1}^{2} c_{k}^{(i)}\left(S_{k}^{(i)}-S_{k 0}^{(i)}-S_{k 0}^{(i)} \ln \frac{S_{k}^{(i)}}{S_{k 0}^{(i)}}\right)+I_{k}\right], \\
L_{2}=\sum_{k=1}^{n} \frac{\omega_{k}}{m_{k}}\left[\sum_{i=1}^{2} \sum_{j=1}^{n} \beta_{k j}^{(i)} \int_{0}^{h} f_{k}^{(i)}(\tau) \int_{t-\tau}^{t} S_{k}^{(i)}(x) I_{j}(x) d x d \tau\right] .
\end{gathered}
$$

Differentiating $L_{1}$ along the solution of system (21), we obtain

$$
\begin{aligned}
\dot{L}_{1}=\sum_{k=1}^{n} \frac{\omega_{k}}{m_{k}}\left\{c_{k}^{(1)}\left[\varphi_{k}\left(S_{k}^{(1)}\right)-\sum_{j=1}^{n} \beta_{k j}^{(1)} S_{k}^{(1)} I_{j}-a_{k} S_{k}^{(1)}\right]\right. \\
+c_{k}^{(2)}\left[a_{k} S_{k}^{(1)}-\sum_{j=1}^{n} \beta_{k j}^{(2)} S_{k}^{(2)} I_{j}-d_{k}^{(2)} S_{k}^{(2)}\right] \\
+\sum_{i=1}^{2} \sum_{j=1}^{n} \int_{0}^{h} f_{k}^{(i)}(\tau) \beta_{k j}^{(i)} S_{k j}^{(i)}(t-\tau) \\
\quad-\frac{c_{k}^{(1)} S_{k 0}^{(1)}}{S_{k}^{(1)}}\left[\varphi_{k}\left(S_{k}^{(1)}\right)-\sum_{j=1}^{n} \beta_{k j}^{(1)} S_{k}^{(1)} I_{j}-a_{k} S_{k}^{(1)}\right] \\
\\
\left.-\frac{c_{k}^{(2)} S_{k 0}^{(2)}}{S_{k}^{(2)}}\left[a_{k} S_{k}^{(1)}-\sum_{j=1}^{n} \beta_{k j}^{(2)} S_{k}^{(2)} I_{j}-d_{k}^{(2)} S_{k}^{(2)}\right]\right\} .
\end{aligned}
$$

Differentiating $L_{2}$ along the solution of system (21), we obtain

$$
\begin{aligned}
L_{2}=\sum_{k=1}^{n} \frac{\omega_{k}}{m_{k}}[ & \sum_{i=1}^{2} \sum_{j=1}^{n} c_{k}^{(i)} \beta_{k j}^{(i)} S_{k}^{(i)} I_{j} \\
& \left.-\sum_{i=1}^{2} \sum_{j=1}^{n} \beta_{k j}^{(i)} \int_{0}^{h} f_{k}^{(i)}(\tau) S_{k}^{(i)}(t-\tau) I_{j}(t-\tau) d \tau\right] .
\end{aligned}
$$

Therefore

$$
\begin{aligned}
& \dot{L}=\sum_{k=1}^{n} \frac{\omega_{k}}{m_{k}}\left\{\varphi_{k}\left(S_{k}^{(1)}\right)\left(c_{k}^{(1)}-\frac{c_{k}^{(1)} S_{k 0}^{(1)}}{S_{k}^{(1)}}\right)+d_{k}^{(2)} S_{k 0}^{(2)}\right. \\
& \times\left(c_{k}^{(2)}-\frac{c_{k}^{(2)} S_{k}^{(2)}}{S_{k 0}^{(2)}}\right)+a_{k} S_{k 0}^{(1)} \\
& \times\left[c_{k}^{(1)}-\frac{c_{k}^{(1)} S_{k}^{(1)}}{S_{k 0}^{(1)}}+\frac{c_{k}^{(2)} S_{k}^{(1)}}{S_{k 0}^{(1)}}-\frac{c_{k}^{(2)} S_{k}^{(1)} S_{k 0}^{(2)}}{S_{k 0}^{(1)} S_{k}^{(2)}}\right] \\
& \left.+\sum_{i=1}^{2} \sum_{j=1}^{n} S_{k 0}^{(i)} c_{k}^{(i)} \beta_{k j}^{(i)} I_{j}-m_{k} I_{k}\right\} \\
& =\sum_{k=1}^{n} \frac{\omega_{k}}{m_{k}}\left\{\varphi_{k}\left(S_{k}^{(1)}\right)\left(c_{k}^{(1)}-\frac{c_{k}^{(1)} S_{k 0}^{(1)}}{S_{k}^{(1)}}\right)\right. \\
& +\sum_{i=1}^{2} \sum_{j=1}^{n} S_{k 0}^{(i)} c_{k}^{(i)} \beta_{k j}^{(i)} I_{j}-m_{k} I_{k} \\
& +d_{k}^{(2)} S_{k 0}^{(2)}\left[c_{k}^{(1)}+c_{k}^{(2)}-\frac{c_{k}^{(1)} S_{k}^{(1)}}{S_{k 0}^{(1)}}+\frac{c_{k}^{(2)} S_{k}^{(1)}}{S_{k 0}^{(1)}}\right. \\
& \left.\left.-\frac{c_{k}^{(2)} S_{k}^{(1)} S_{k 0}^{(2)}}{S_{k 0}^{(1)} S_{k}^{(2)}}-\frac{c_{k}^{(2)} S_{k}^{(2)}}{S_{k 0}^{(2)}}\right]\right\} .
\end{aligned}
$$

From (23), we know that

$$
\begin{array}{r}
\varphi_{k}\left(S_{k 0}^{(1)}\right)\left(c_{k}^{(1)}-\frac{c_{k}^{(1)} S_{k 0}^{(1)}}{S_{k}^{(1)}}\right)=d_{k}^{(2)} S_{k 0}^{(2)}\left(c_{k}^{(1)}-\frac{c_{k}^{(1)} S_{k 0}^{(1)}}{S_{k}^{(1)}}\right), \\
k=1,2, \ldots, n .
\end{array}
$$

By (45), we obtain

$$
\begin{aligned}
\dot{L}=\sum_{k=1}^{n} \frac{\omega_{k}}{m_{k}}\{[ & \left.\varphi_{k}\left(S_{k}^{(1)}\right)-\varphi_{k}\left(S_{k 0}^{(1)}\right)\right]\left(c_{k}^{(1)}-\frac{c_{k}^{(1)} S_{k 0}^{(1)}}{S_{k}^{(1)}}\right) \\
& +\sum_{i=1}^{2} \sum_{j=1}^{n} S_{k 0}^{(i)} c_{k}^{(i)} \beta_{k j}^{(i)} I_{j}-m_{k} I_{k} \\
+ & d_{k}^{(2)} S_{k 0}^{(2)}\left[2 c_{k}^{(1)}+c_{k}^{(2)}-\frac{c_{k}^{(1)} S_{k}^{(1)}}{S_{k 0}^{(1)}}-\frac{c_{k}^{(1)} S_{k 0}^{(1)}}{S_{k}^{(1)}}\right. \\
& \left.\left.+\frac{c_{k}^{(2)} S_{k}^{(1)}}{S_{k 0}^{(1)}}-\frac{c_{k}^{(2)} S_{k}^{(1)} S_{k 0}^{(2)}}{S_{k 0}^{(1)} S_{k}^{(2)}}-\frac{c_{k}^{(2)} S_{k}^{(2)}}{S_{k 0}^{(2)}}\right]\right\} .
\end{aligned}
$$


We can rewrite the equation as

$$
\begin{aligned}
\dot{L}=\sum_{k=1}^{n} \frac{\omega_{k}}{m_{k}}\left\{\left[\varphi_{k}\left(S_{k}^{(1)}\right)-\varphi_{k}\left(S_{k 0}^{(1)}\right)\right]\left(c_{k}^{(1)}-\frac{c_{k}^{(1)} S_{k 0}^{(1)}}{S_{k}^{(1)}}\right)\right. \\
+\sum_{i=1}^{2} \sum_{j=1}^{n} S_{k 0}^{(i)} c_{k}^{(i)} \beta_{k j}^{(i)} I_{j}-m_{k} I_{k} \\
+d_{k}^{(2)} S_{k 0}^{(2)}\left[2 c_{k}^{(1)}+c_{k}^{(2)}-\left(c_{k}^{(1)}-c_{k}^{(2)}\right)\right. \\
\quad \times\left(\frac{S_{k}^{(1)}}{S_{k 0}^{(1)}}+\frac{S_{k 0}^{(1)}}{S_{k}^{(1)}}\right) \\
\left.\left.\quad-c_{k}^{(2)}\left(\frac{S_{k 0}^{(1)}}{S_{k}^{(1)}}+\frac{S_{k}^{(1)} S_{k 0}^{(2)}}{S_{k 0}^{(1)} S_{k}^{(2)}}+\frac{S_{k}^{(2)}}{S_{k 0}^{(2)}}\right)\right]\right\} .
\end{aligned}
$$

By the fact that $\varphi_{k}$ is strictly decreasing function and the arithmetic-geometric mean, we have

$$
\begin{aligned}
\dot{L} \leq \sum_{k=1}^{n} \frac{\omega_{k}}{m_{k}}\left\{\sum_{i=1}^{2} \sum_{j=1}^{n} S_{k 0}^{(i)} c_{k}^{(i)} \beta_{k j}^{(i)} I_{j}-m_{k} I_{k}+d_{k}^{(2)} S_{k 0}^{(2)}\right. \\
\left.\times\left[2 c_{k}^{(1)}+c_{k}^{(2)}-2\left(c_{k}^{(1)}-c_{k}^{(2)}\right)-3 c_{k}^{(2)}\right]\right\} \\
=\sum_{k=1}^{n} \frac{\omega_{k}}{m_{k}}\left(\sum_{i=1}^{2} \sum_{j=1}^{n} S_{k 0}^{(i)} c_{k}^{(i)} \beta_{k j}^{(i)} I_{j}-m_{k} I_{k}\right)=: U
\end{aligned}
$$

where equality holds if and only if

$$
S_{k}^{(1)}=S_{k 0}^{(1)}, \quad S_{k}^{(2)}=S_{k 0}^{(2)}, \quad k=1,2, \ldots, n .
$$

Thus

$$
\begin{aligned}
U & =\sum_{k=1}^{n} \omega_{k}\left[\frac{\sum_{i=1}^{2} c_{k}^{(i)} \sum_{j=1}^{n} \beta_{k j}^{(i)} S_{k 0}^{(i)} I_{j}}{m_{k}}-I_{k}\right] \\
& =\left(\omega_{1}, \omega_{2}, \ldots, \omega_{n}\right)\left(\mathbf{Q I} \mathbf{I}^{T}-\mathbf{I}^{T}\right) .
\end{aligned}
$$

If $R_{0}<1$, then $\dot{L}=0$ if and only if $\mathbf{I}^{T}=\mathbf{0}$. If $R_{0}=1$, then $\dot{L}=0$ implies $U=0$. If $R_{0}=1$ and $\dot{U}=0$, then (49) holds. If (49) holds, then, from the first two equations of (21), we may obtain $\sum_{j=1}^{n} \beta_{k j}^{(1)} S_{k}^{(1)} I_{j} \equiv 0, \sum_{j=1}^{n} \beta_{k j}^{(2)} S_{k}^{(2)} I_{j} \equiv 0, k=1,2 \ldots, n$. Therefore, if $R_{0}=1$ and $\dot{U}=0$, then we have $I_{k}=0$. Therefore, $\dot{L}=0$ if and only if $\mathbf{I}=\mathbf{0}$ and $\mathbf{S}=\mathbf{S}^{\mathbf{0}}$. Hence the largest invariant subset of the set where $\dot{L}=0$ is the singleton $\left\{P_{0}\right\}$. By LaSalle's Invariance Principle, $P_{0}$ is globally attractive. Using the same proof as the one for Corollary 5.3.1 in [16], we can show that $P_{0}$ is locally stable. Hence, the diseasefree equilibrium $P_{0}$ is globally asymptotically stable in $\Gamma$ for $R_{0} \leq 1$. This completes the proof.
Theorem 8. Under Assumption 1, $P^{*}$ of system (21) is globally asymptotically stable in $\Gamma$, if $c_{k}^{(1)} \geq c_{k}^{(2)}, k=1,2, \ldots, n$, and $R_{0}>1$.

Proof. Set $\bar{\beta}_{k j}=\sum_{i=1}^{2} c_{k}^{(i)} \beta_{k j}^{(i)} S_{k}^{(i) *} I_{j}^{*}, 1 \leq k, j \leq n$. Consider a Lyapunov functional $V=V_{1}+V_{2}$, where

$$
\begin{gathered}
V_{1}=\sum_{k=1}^{n} v_{k}\left[\sum_{i=1}^{2} c_{k}^{(i)}\left(S_{k}^{(i)}-S_{k}^{(i) *}-S_{k}^{(i) *} \ln \frac{S_{k}^{(i)}}{S_{k}^{(i) *}}\right)\right. \\
\left.\quad+I_{k}-I_{k}^{*}-I_{k}^{*} \ln \frac{I_{k}}{I_{k}^{*}}\right], \\
V_{2}=\sum_{k=1}^{n} v_{k} \sum_{i=1}^{2} \sum_{j=1}^{n} \beta_{k j}^{(i)} \\
\quad \times \int_{0}^{h} f_{k}^{(i)}(\tau) \int_{t-\tau}^{t}\left(\begin{array}{c}
S_{k}^{(i)}(x) I_{j}(x)-S_{k}^{(i) *} I_{j}^{*}-S_{k}^{(i) *} I_{j}^{*} \\
\left.\times \ln \frac{S_{k}^{(i)}(x) I_{j}(x)}{S_{k}^{(i) *} I_{j}^{*}}\right) d x d \tau .
\end{array}\right.
\end{gathered}
$$

Differentiating $V_{1}$ along the solution of system (21), we obtain

$$
\begin{aligned}
\dot{V}_{1}=\sum_{k=1}^{n} v_{k}\left\{c_{k}^{(1)}\left[\varphi_{k}\left(S_{k}^{(1)}\right)-\sum_{j=1}^{n} \beta_{k j}^{(1)} S_{k}^{(1)} I_{j}-a_{k} S_{k}^{(1)}\right]\right. \\
+c_{k}^{(2)}\left[a_{k} S_{k}^{(1)}-\sum_{j=1}^{n} \beta_{k j}^{(2)} S_{k}^{(2)} I_{j}-d_{k}^{(2)} S_{k}^{(2)}\right] \\
+\sum_{i=1}^{2} \sum_{j=1}^{n} \int_{0}^{h} f_{k}^{(i)}(\tau) \beta_{k j}^{(i)} S_{k}^{(i)}(t-\tau) I_{j}(t-\tau) d \tau \\
-\frac{c_{k}^{(1)} S_{k}^{(1) *}}{S_{k}^{(1)}}\left[\varphi_{k}\left(S_{k}^{(1)}\right)-\sum_{j=1}^{n} \beta_{k j}^{(1)} S_{k}^{(1)} I_{j}-a_{k} S_{k}^{(1)}\right. \\
-\frac{c_{k}^{(2)} S_{k}^{(2) *}}{S_{k}^{(2)}}\left[a_{k} S_{k}^{(1)}-\sum_{j=1}^{n} \beta_{k j}^{(2)} S_{k}^{(2)} I_{j}-d_{k}^{(2)} S_{k}^{(2)}\right] \\
-\frac{I_{k}^{*}\left[\sum_{i=1}^{2} \sum_{j=1}^{n} \int_{0}^{h} f_{k}^{(i)}(\tau) \beta_{k j}^{(i)} S_{k}^{(i)}(t-\tau)\right.}{\left.\left.I_{k} I_{j}(t-\tau) d \tau-m_{k} I_{k}\right]\right\} .} \\
\\
+
\end{aligned}
$$


Differentiating $V_{2}$ along the solution of system (21), we obtain

$$
\begin{aligned}
\dot{V}_{2}= & \sum_{k=1}^{n} v_{k} \sum_{i=1}^{2} \sum_{j=1}^{n} \beta_{k j}^{(i)} \\
& \times\left\{c_{k}^{(i)} S_{k}^{(i)} I_{j}\right. \\
& -\int_{0}^{h} f_{k}^{(i)}(\tau)\left[S_{k}^{(i)}(t-\tau) I_{j}(t-\tau)+S_{k}^{(i) *} I_{j}^{*}\right. \\
& \left.\left.\quad \times \ln \frac{S_{k}^{(i)} I_{j}}{S_{k}^{(i)}(t-\tau) I_{j}(t-\tau)}\right] d \tau\right\} .
\end{aligned}
$$

Therefore

$$
\begin{aligned}
& \dot{V}=\sum_{k=1}^{n} v_{k}\left\{\varphi_{k}\left(S_{k}^{(1)}\right)\left(c_{k}^{(1)}-\frac{c_{k}^{(1)} S_{k}^{(1) *}}{S_{k}^{(1)}}\right)\right. \\
& +d_{k}^{(2)} S_{k}^{(2) *}\left(c_{k}^{(2)}-\frac{c_{k}^{(2)} S_{k}^{(2)}}{S_{k}^{(2) *}}\right)+\sum_{i=1}^{2} c_{k}^{(i)} \sum_{j=1}^{n} \beta_{k j}^{(i)} S_{k}^{(i) *} I_{j} \\
& +m_{k} I_{k}^{*}-m_{k} I_{k}+a_{k} S_{k}^{(1) *} \\
& \times\left[c_{k}^{(1)}-\frac{c_{k}^{(1)} S_{k}^{(1)}}{S_{k}^{(1) *}}+\frac{c_{k}^{(2)} S_{k}^{(1)}}{S_{k}^{(1) *}}-\frac{c_{k}^{(2)} S_{k}^{(1)} S_{k}^{(2) *}}{S_{k}^{(1) *} S_{k}^{(2)}}\right] \\
& -\frac{I_{k}^{*}}{I_{k}}\left[\sum_{i=1}^{2} \sum_{j=1}^{n} \int_{0}^{h} f_{k}^{(i)}(\tau) \beta_{k j}^{(i)} S_{k}^{(i)}\right. \\
& \left.\left.\times(t-\tau) I_{j}(t-\tau) d \tau\right]-\Delta\right\},
\end{aligned}
$$

where

$$
\Delta=\sum_{i=1}^{2} \sum_{j=1}^{n} \beta_{k j}^{(i)} \int_{0}^{h} f_{k}^{(i)}(\tau) S_{k}^{(i) *} I_{j}^{*} \ln \frac{S_{k}^{(i)} I_{j}}{S_{k}^{(i)}(t-\tau) I_{j}(t-\tau)} d \tau
$$

From (38), we know that

$$
\begin{aligned}
\varphi_{k}\left(S_{k}^{(1) *}\right) & \left(c_{k}^{(1)}-\frac{c_{k}^{(1)} S_{k}^{(1) *}}{S_{k}^{(1)}}\right) \\
= & \left(\sum_{i=1}^{2} \sum_{j=1}^{n} \beta_{k j}^{(i)} S_{k}^{(i) *} I_{j}^{*}+d_{k}^{(2)} S_{k}^{(2) *}\right)\left(c_{k}^{(1)}-\frac{c_{k}^{(1)} S_{k}^{(1) *}}{S_{k}^{(1)}}\right) .
\end{aligned}
$$

It follows from (39), (40), and (56) that

$$
\begin{aligned}
& \dot{V}=\sum_{k=1}^{n} v_{k}\left\{\left[\varphi_{k}\left(S_{k}^{(1)}\right)-\varphi_{k}\left(S_{k}^{(1) *}\right)\right]\left(c_{k}^{(1)}-\frac{c_{k}^{(1)} S_{k}^{(1) *}}{S_{k}^{(1)}}\right)\right. \\
& +d_{k}^{(2)} S_{k}^{(2) *}\left(c_{k}^{(2)}-\frac{c_{k}^{(2)} S_{k}^{(2)}}{S_{k}^{(2) *}}\right) \\
& +\sum_{i=1}^{2} c_{k}^{(i)} \beta_{k j}^{(i)} S_{k}^{(i) *} I_{j}^{*}\left(\frac{I_{j}}{I_{j}^{*}}-\frac{I_{k}}{I_{k}^{*}}+1\right) \\
& +S_{k}^{(1) *} \sum_{j=1}^{n} \beta_{k j}^{(1)} I_{j}^{*}\left(c_{k}^{(1)}-\frac{c_{k}^{(1)} S_{k}^{(1) *}}{S_{k}^{(1)}}\right) \\
& +\left(d_{k}^{(2)} S_{k}^{(2) *}+S_{k}^{(2) *} \sum_{j=1}^{n} \beta_{k j}^{(2)} I_{j}^{*}\right) \\
& \times\left[2 c_{k}^{(1)}-\frac{c_{k}^{(1)} S_{k}^{(1) *}}{S_{k}^{(1)}}-\frac{c_{k}^{(1)} S_{k}^{(1)}}{S_{k}^{(1) *}}\right. \\
& \left.+\frac{c_{k}^{(2)} S_{k}^{(1)}}{S_{k}^{(1) *}}-\frac{c_{k}^{(2)} S_{k}^{(1)} S_{k}^{(2) *}}{S_{k}^{(1) *} S_{k}^{(2)}}\right]-\frac{I_{k}^{*}}{I_{k}} \\
& \times\left[\sum_{i=1}^{2} \sum_{j=1}^{n} \int_{0}^{h} f_{k}^{(i)}(\tau) \beta_{k j}^{(i)} S_{k}^{(i)}(t-\tau) I_{j}(t-\tau) d \tau\right] \\
& -\Delta\} \text {. }
\end{aligned}
$$

By Theorem 2 and the fact that $\varphi_{k}$ is strictly decreasing function, we obtain

$$
\begin{aligned}
\dot{V} \leq \sum_{k=1}^{n} v_{k}\{ & d_{k}^{(2)} S_{k}^{(2) *}\left(c_{k}^{(2)}-\frac{c_{k}^{(2)} S_{k}^{(2)}}{S_{k}^{(2) *}}\right) \\
& +\sum_{i=1}^{2} c_{k}^{(i)} \beta_{k j}^{(i)} S_{k}^{(i) *} I_{j}^{*} \\
& +S_{k}^{(1) *} \sum_{j=1}^{n} \beta_{k j}^{(1)} I_{j}^{*}\left(c_{k}^{(1)}-\frac{c_{k}^{(1)} S_{k}^{(1) *}}{S_{k}^{(1)}}\right) \\
& +\left(d_{k}^{(2)} S_{k}^{(2) *}+S_{k}^{(2) *} \sum_{j=1}^{n} \beta_{k j}^{(2)} I_{j}^{*}\right) \\
& \times\left[2 c_{k}^{(1)}-\frac{c_{k}^{(1)} S_{k}^{(1) *}}{S_{k}^{(1)}}-\frac{c_{k}^{(1)} S_{k}^{(1)}}{S_{k}^{(1) *}}\right. \\
& \left.+\frac{c_{k}^{(2)} S_{k}^{(1)}}{S_{k}^{(1) *}}-\frac{c_{k}^{(2)} S_{k}^{(1)} S_{k}^{(2) *}}{S_{k}^{(1) *} S_{k}^{(2)}}\right]
\end{aligned}
$$




$$
\begin{aligned}
& -\frac{I_{k}^{*}}{I_{k}}\left[\sum_{i=1}^{2} \sum_{j=1}^{n} \int_{0}^{h} f_{k}^{(i)}(\tau) \beta_{k j}^{(i)} S_{k}^{(i)}\right. \\
& \left.\left.\times(t-\tau) I_{j}(t-\tau) d \tau\right]-\Delta\right\} \\
& =\sum_{k=1}^{n} v_{k}\left\{d _ { k } ^ { ( 2 ) } S _ { k } ^ { ( 2 ) * } \left(c_{k}^{(2)}+2 c_{k}^{(1)}-\frac{c_{k}^{(1)} S_{k}^{(1) *}}{S_{k}^{(1)}}-\frac{c_{k}^{(1)} S_{k}^{(1)}}{S_{k}^{(1) *}}\right.\right. \\
& \left.+\frac{c_{k}^{(2)} S_{k}^{(1)}}{S_{k}^{(1) *}}-\frac{c_{k}^{(2)} S_{k}^{(1)} S_{k}^{(2) *}}{S_{k}^{(1) *} S_{k}^{(2)}}-\frac{c_{k}^{(2)} S_{k}^{(2)}}{S_{k}^{(2) *}}\right) \\
& +S_{k}^{(2) *} \sum_{j=1}^{n} \beta_{k j}^{(2)} I_{j}^{*}\left[2 c_{k}^{(1)}+c_{k}^{(2)}-\frac{c_{k}^{(1)} S_{k}^{(1) *}}{S_{k}^{(1)}}-\frac{c_{k}^{(1)} S_{k}^{(1)}}{S_{k}^{(1) *}}\right. \\
& \left.+\frac{c_{k}^{(2)} S_{k}^{(1)}}{S_{k}^{(1) *}}-\frac{c_{k}^{(2)} S_{k}^{(1)} S_{k}^{(2) *}}{S_{k}^{(1) *} S_{k}^{(2)}}\right] \\
& +S_{k}^{(1) *} \sum_{j=1}^{n} \beta_{k j}^{(1)} I_{j}^{*}\left(2 c_{k}^{(1)}-\frac{c_{k}^{(1)} S_{k}^{(1) *}}{S_{k}^{(1)}}\right) \\
& -\frac{I_{k}^{*}}{I_{k}}\left[\sum_{i=1}^{2} \sum_{j=1}^{n} \int_{0}^{h} f_{k}^{(i)}(\tau) \beta_{k j}^{(i)} S_{k}^{(i)}\right. \\
& \left.\left.\times(t-\tau) I_{j}(t-\tau) d \tau\right]-\Delta\right\} \\
& =\sum_{k=1}^{n} v_{k}\left\{d _ { k } ^ { ( 2 ) } S _ { k } ^ { ( 2 ) * } \left[c_{k}^{(2)}+2 c_{k}^{(1)}-\left(c_{k}^{(1)}-c_{k}^{(2)}\right)\right.\right. \\
& \times\left(\frac{S_{k}^{(1) *}}{S_{k}^{(1)}}+\frac{S_{k}^{(1)}}{S_{k}^{(1) *}}\right) \\
& \left.-c_{k}^{(2)}\left(\frac{S_{k}^{(1) *}}{S_{k}^{(1)}}+\frac{S_{k}^{(1)} S_{k}^{(2) *}}{S_{k}^{(1) *} S_{k}^{(2)}}+\frac{S_{k}^{(2)}}{S_{k}^{(2) *}}\right)\right]+S_{k}^{(2) *} \\
& \times \sum_{j=1}^{n} \beta_{k j}^{(2)} I_{j}^{*}\left[2 c_{k}^{(1)}+c_{k}^{(2)}-\left(c_{k}^{(1)}-c_{k}^{(2)}\right)\right. \\
& \times\left(\frac{S_{k}^{(1) *}}{S_{k}^{(1)}}+\frac{S_{k}^{(1)}}{S_{k}^{(1) *}}\right) \\
& -\int_{0}^{h} f_{k}^{(2)}(\tau) \\
& \times\left(\frac{S_{k}^{(1) *}}{S_{k}^{(1)}}+\frac{S_{k}^{(1)} S_{k}^{(2) *}}{S_{k}^{(1) *} S_{k}^{(2)}}\right. \\
& +\left(\left(S_{k}^{(2)} I_{k}^{*} S_{k}^{(2)}\right.\right. \\
& \left.\times(t-\tau) I_{j}(t-\tau)\right) \\
& \left.\left.\left.\times\left(S_{k}^{(2) *} S_{k}^{(2)} I_{k} I_{j}^{*}\right)^{-1}\right)\right) d \tau\right] \\
& \times\left[2 c_{k}^{(1)}\right. \\
& -\int_{0}^{h} f_{k}^{(1)}(\tau) \\
& \times\left(\frac{S_{k}^{(1) *}}{S_{k}^{(1)}}\right. \\
& +\left(\left(S_{k}^{(1)} I_{k}^{*} S_{k}^{(1)}(t-\tau) I_{j}(t-\tau)\right)\right. \\
& \left.\left.\left.\times\left(S_{k}^{(1) *} S_{k}^{(1)} I_{k} I_{j}^{*}\right)^{-1}\right)\right) d \tau\right] \\
& -\Delta\}=: B_{1} . \\
& B_{1} \leq \sum_{k=1}^{n} v_{k}\left\{d_{k}^{(2)} S_{k}^{(2) *}\left[c_{k}^{(2)}+2 c_{k}^{(1)}-2\left(c_{k}^{(1)}-c_{k}^{(2)}\right)-3 c_{k}^{(2)}\right]\right. \\
& +S_{k}^{(2) *} \sum_{j=1}^{n} \beta_{k j}^{(2)} I_{j}^{*}\left[2 c_{k}^{(1)}+c_{k}^{(2)}-2\left(c_{k}^{(1)}-c_{k}^{(2)}\right)\right. \\
& -3 \int_{0}^{h} f_{k}^{(2)}(\tau) \\
& \left.\times\left(\frac{I_{k}^{*} S_{k}^{(2)}(t-\tau) I_{j}(t-\tau)}{S_{k}^{(2)} I_{k} I_{j}^{*}}\right)^{1 / 3} d \tau\right] \\
& +S_{k}^{(1) *} \sum_{j=1}^{n} \beta_{k j}^{(1)} I_{j}^{*} \\
& \times\left[2 c_{k}^{(1)}\right. \\
& -2 \int_{0}^{h} f_{k}^{(1)}(\tau) \\
& \left.\times\left(\frac{I_{k}^{*} S_{k}^{(1)}(t-\tau) I_{j}(t-\tau)}{S_{k}^{(1)} I_{k} I_{j}^{*}}\right)^{1 / 2} d \tau\right] \\
& -\Delta\} \\
& =\sum_{k=1}^{n} v_{k}\left\{3 S_{k}^{(2) *} \sum_{j=1}^{n} \beta_{k j}^{(2)} I_{j}^{*}\right. \\
& \times\left[c_{k}^{(2)}\right. \\
& +S_{k}^{(1) *} \sum_{j=1}^{n} \beta_{k j}^{(1)} I_{j}^{*} \\
& -\int_{0}^{h} f_{k}^{(2)}(\tau)
\end{aligned}
$$




$$
\begin{array}{r}
\left.\times\left(\frac{I_{k}^{*} S_{k}^{(2)}(t-\tau) I_{j}(t-\tau)}{S_{k}^{(2)} I_{k} I_{j}^{*}}\right)^{1 / 3} d \tau\right] \\
+2 S_{k}^{(1) *} \\
\times \sum_{j=1}^{n} \beta_{k j}^{(1)} I_{j}^{*}\left[c_{k}^{(1)}\right. \\
\quad-\int_{0}^{h} f_{k}^{(1)}(\tau) \\
\left.\quad \times\left(\frac{I_{k}^{*} S_{k}^{(1)}(t-\tau) I_{j}(t-\tau)}{S_{k}^{(1)} I_{k} I_{j}^{*}}\right)^{1 / 2} d \tau\right] \\
-\Delta\}=: B_{2} .
\end{array}
$$

Let $Y(x)=1-x+\ln x$. We can rewrite $B_{2}$ as

$$
\begin{aligned}
\sum_{k=1}^{n} v_{k}\left\{3 S_{k}^{(2) *} \sum_{j=1}^{n} \beta_{k j}^{(2)} I_{j}^{*}\right. & \\
& \times \int_{0}^{h} f_{k}^{(2)}(\tau) Y \\
& \times\left(\left[\frac{I_{k}^{*} S_{k}^{(2)}(t-\tau) I_{j}(t-\tau)}{S_{k}^{(2)} I_{k} I_{j}^{*}}\right]^{1 / 3}\right) d \tau \\
& +2 S_{k}^{(1) *} \sum_{j=1}^{n} \beta_{k j}^{(1)} I_{j}^{*} \\
& \times \int_{0}^{h} f_{k}^{(1)}(\tau) Y \\
& \times\left(\left[\frac{I_{k}^{*} S_{k}^{(1)}(t-\tau) I_{j}(t-\tau)}{S_{k}^{(1)} I_{k} I_{j}^{*}}\right]^{1 / 2}\right) d \tau \\
& -\sum_{i=1}^{2} \sum_{j=1}^{n} \beta_{k j}^{(i)} \\
& \times \int_{0}^{h} f_{k}^{(i)}(\tau) S_{k}^{(i) *} I_{j}^{*} \\
& \left.\times \ln \frac{I_{k}^{*} S_{k}^{(i)}(t-\tau) I_{j}(t-\tau)}{I_{k} I_{j}^{*} S_{k}^{(i)}} d \tau-\Delta\right\} .
\end{aligned}
$$

By Theorem 2 and the fact that $Y(x) \leq 0$, where equality holds if and only if $x=1$, we obtain

$$
\begin{aligned}
B_{2} \leq \sum_{k=1}^{n} v_{k}\left\{-\sum_{i=1}^{2} \sum_{j=1}^{n} \beta_{k j}^{(i)} \int_{0}^{h} f_{k}^{(i)}(\tau) S_{k}^{(i) *} I_{j}^{*}\right. \\
\left.\times \ln \frac{I_{k}^{*} S_{k}^{(i)}(t-\tau) I_{j}(t-\tau)}{I_{k} I_{j}^{*} S_{k}^{(i)}} d \tau-\Delta\right\} \\
=-\sum_{k=1}^{n} v_{k} \sum_{i=1}^{2} \sum_{j=1}^{n} c_{k}^{(i)} \beta_{k j}^{(i)} S_{k}^{(i) *} I_{j}^{*} \ln \frac{I_{k}^{*} I_{j}}{I_{k} I_{j}^{*}} \\
=-\sum_{k, j=1}^{n} v_{k} \bar{\beta}_{k j} \ln \frac{I_{k}^{*} I_{j}}{I_{k} I_{j}^{*}}=0 .
\end{aligned}
$$

From (58) and (59), we see that if $\dot{V}=0$, then

$$
S_{k}^{(i)}=S_{k}^{(i) *}, \quad i=1,2, k=1,2, \ldots, n .
$$

If (62) holds, it follows from (21) that

$$
\begin{gathered}
0=\varphi_{k}\left(S_{k}^{(1) *}\right)-\sum_{j=1}^{n} \beta_{k j}^{(1)} S_{k}^{(1) *} I_{j}-a_{k} S_{k}^{(1) *}, \\
0=a_{k} S_{k}^{(1) *}-\sum_{j=1}^{n} \beta_{k j}^{(2)} S_{k}^{(2) *} I_{j}-d_{k}^{(2)} S_{k}^{(2) *}, \quad k=1,2, \ldots, n .
\end{gathered}
$$

Then, we obtain that

$$
\begin{gathered}
\sum_{j=1}^{n} \beta_{k j}^{(1)} S_{k}^{(1) *} I_{j}=\varphi_{k}\left(S_{k}^{(1) *}\right)-a_{k} S_{k}^{(1) *}, \\
\sum_{j=1}^{n} \beta_{k j}^{(2)} S_{k}^{(2) *} I_{j}=a_{k} S_{k}^{(1) *}-d_{k}^{(2)} S_{k}^{(2) *}, \quad k=1,2, \ldots, n .
\end{gathered}
$$

Thus,

$$
\begin{array}{r}
\dot{I}_{k}=c_{k}^{(1)}\left(\varphi_{k}\left(S_{k}^{(1) *}\right)-a_{k} S_{k}^{(1) *}\right) \\
+c_{k}^{(2)}\left(a_{k} S_{k}^{(1) *}-d_{k}^{(2)} S_{k}^{(2) *}\right)-m_{k} I_{k}, \\
k=1,2, \ldots, n .
\end{array}
$$

This implies that

$$
\begin{aligned}
\lim _{t \rightarrow+\infty} I_{k} & \\
& =\frac{c_{k}^{(1)}\left(\varphi_{k}\left(S_{k}^{(1) *}\right)-a_{k} S_{k}^{(1) *}\right)+c_{k}^{(2)}\left(a_{k} S_{k}^{(1) *}-d_{k}^{(2)} S_{k}^{(2) *}\right)}{m_{k}} \\
& =I_{k}^{*} .
\end{aligned}
$$

Hence, the largest invariant subset of the set where $\dot{V}=0$ is the singleton $\left\{P^{*}\right\}$. By LaSalle's Invariance Principle, $P^{*}$ is 
globally attractive. By a similar argument to that in the proof of Theorem $7, P^{*}$ is globally asymptotically stable in $\Gamma$ for $R_{0}>1$.

Following [17], we set matrices

$$
\mathbf{F}:=\left(\sum_{i=1}^{2} c_{k}^{(i)} \beta_{k j}^{(i)} S_{k 0}^{(i)}\right)_{n \times n}, \quad \mathbf{V}:=\operatorname{diag}\left(m_{1}, m_{2}, \ldots, m_{n}\right) .
$$

The next generation matrix for system (16) is

$$
\mathbf{Q}:=\mathbf{F V}^{-\mathbf{1}}=\left(\frac{\sum_{i=1}^{2} c_{k}^{(i)} \beta_{k j}^{(i)} S_{k 0}^{(i)}}{m_{k}}\right)_{n \times n} .
$$

Let $r_{0}=\rho(\mathbf{Q})$, where $\rho$ denotes the spectral radius.

By Lemma 6 and Theorems 2, 4, 7, and 8, we can obtain the following results.

Corollary 9. Assume that $\mathbf{B}=\left[\sum_{i=1}^{2} \beta_{k j}^{(i)}\right]$ is irreducible and $c_{k}^{(1)} \geq c_{k}^{(2)}, k=1,2, \ldots, n$. If $r_{0} \leq 1$, then $P_{0}$ of system (21) is globally asymptotically stable in $\Gamma$.

Corollary 10. Assume that $\mathbf{B}=\left[\sum_{i=1}^{2} \beta_{k j}^{(i)}\right]$ is irreducible and $c_{k}^{(1)} \geq c_{k}^{(2)}, k=1,2, \ldots, n$. If $r_{0}>1$, then $P^{*}$ of system (21) is globally asymptotically stable in $\Gamma$.

Remark 11. Note that, for Theorems 7 and 8, we do not assume that $\mathbf{B}=\left[\sum_{i=1}^{2} \beta_{k j}^{(i)}\right]$ is irreducible. If Theorems 7 and 8 hold, then Corollaries 9 and 10 hold too, respectively. However, it is easy to show some examples which conform to the conditions of Theorem 7 or Theorem 8 but are not in accord with the conditions of Corollary 9 or Corollary 10. It seems that all authors use the the graph theory under the assumption that the relative matrix is irreducible which is analogous with the conditions of Corollaries 9 and 10 (see, e.g., [1-13]).

\section{Acknowledgments}

This work was supported by the National Basic Research Program of China (2010CB732501), and the National Natural Science Foundation of China (61273015).

\section{References}

[1] H. Guo, M. Y. Li, and Z. Shuai, "Global stability of the endemic equilibrium of multigroup SIR epidemic models," Canadian Applied Mathematics Quarterly, vol. 14, no. 3, pp. 259-284, 2006.

[2] H. Guo, M. Y. Li, and Z. Shuai, "A graph-theoretic approach to the method of global Lyapunov functions," Proceedings of the American Mathematical Society, vol. 136, no. 8, pp. 2793-2802, 2008.

[3] M. Y. Li and Z. Shuai, "Global-stability problem for coupled systems of differential equations on networks," Journal of Differential Equations, vol. 248, no. 1, pp. 1-20, 2010.

[4] H. Su, W. Li, and K. Wang, "Global stability of discrete-time coupled systems on networks and its applications," Chaos, vol. 22, Article ID 033135, 2012.
[5] C. Zhang, W. Li, and K. Wang, "Boundedness for network of stochastic coupled van der Pol oscillators with time-varying delayed coupling," Applied Mathematical Modelling. Simulation and Computation for Engineering and Environmental Systems, vol. 37, no. 7, pp. 5394-5402, 2013.

[6] W. Li, H. Su, D. Wei, and K. Wang, "Global stability of coupled nonlinear systems with Markovian switching," Communications in Nonlinear Science and Numerical Simulation, vol. 17, no. 6, pp. 2609-2616, 2012.

[7] C. Ji, D. Jiang, and N. Shi, "Multigroup SIR epidemic model with stochastic perturbation," Physica A, vol. 390, pp. 1747-1762, 2011.

[8] Q. Yang and X. Mao, "Extinction and recurrence of multigroup SEIR epidemic models with stochastic perturbations," Nonlinear Analysis: Real World Applications, vol. 11, pp. 9951004, 2010.

[9] T. Kuniya, "Global stability analysis with a discretization approach for an age-structured multigroup SIR epidemic model," Nonlinear Analysis: Real World Applications, vol. 12, no. 5, pp. 2640-2655, 2011.

[10] Z. Yuan and X. Zou, "Global threshold property in an epidemic model for disease with latency spreading in a heterogeneous host population," Nonlinear Analysis: Real World Applications, vol. 11, no. 5, pp. 3479-3490, 2010.

[11] M. Y. Li, Z. Shuai, and C. Wang, "Global stability of multigroup epidemic models with distributed delays," Journal of Mathematical Analysis and Applications, vol. 361, no. 1, pp. 3847, 2010.

[12] Y. Muroya, Y. Enatsu, and T. Kuniya, "Global stability for a multi-group SIRS epidemic model with varying population sizes," Nonlinear Analysis: Real World Applications, vol. 14, no. 3, pp. 1693-1704, 2013.

[13] "A multi-group sveir epidemic model with distributed delay and vaccination," International Journal of Biomathematics, vol. 5, pp. 1-18, 2012.

[14] H. R. Thieme, "Global stability of the endemic equilibrium in infinite dimension: Lyapunov functions and positive operators," Journal of Differential Equations, vol. 250, no. 9, pp. 3772-3801, 2011.

[15] H. I. Freedman, S. G. Ruan, and M. X. Tang, "Uniform persistence and flows near a closed positively invariant set," Journal of Dynamics and Differential Equations, vol. 6, no. 4, pp. 583-600, 1994

[16] J. K. Hale and S. M. Verduyn Lunel, Introduction to FunctionalDifferential Equations, vol. 99, Springer, New York, NY, USA, 1993.

[17] P. van den Driessche and J. Watmough, "Reproduction numbers and sub-threshold endemic equilibria for compartmental models of disease transmission," Mathematical Biosciences, vol. 180, pp. $29-48,2002$. 


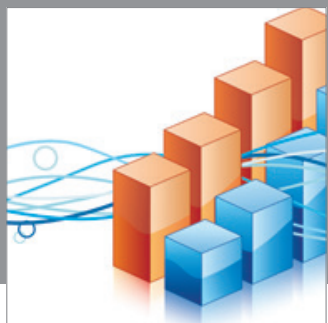

Advances in

Operations Research

mansans

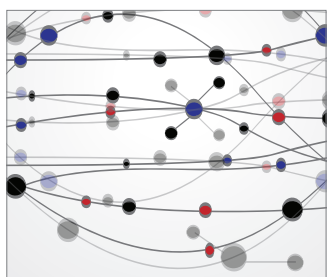

The Scientific World Journal
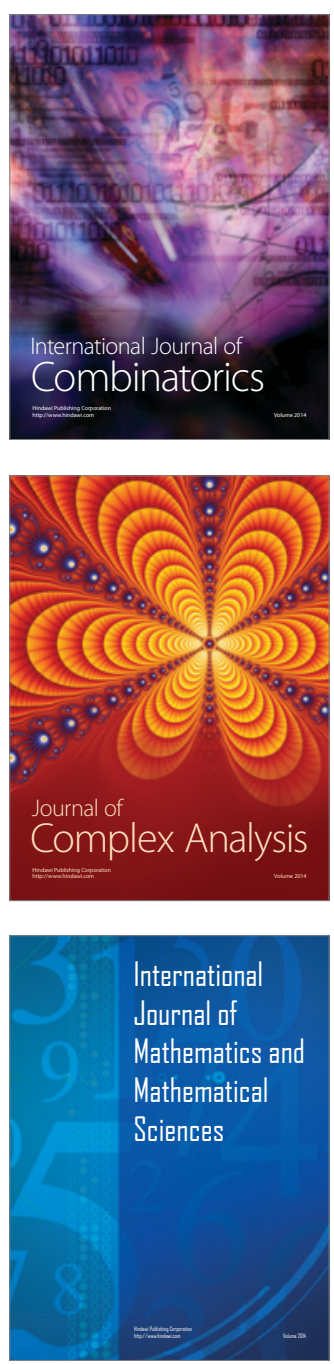
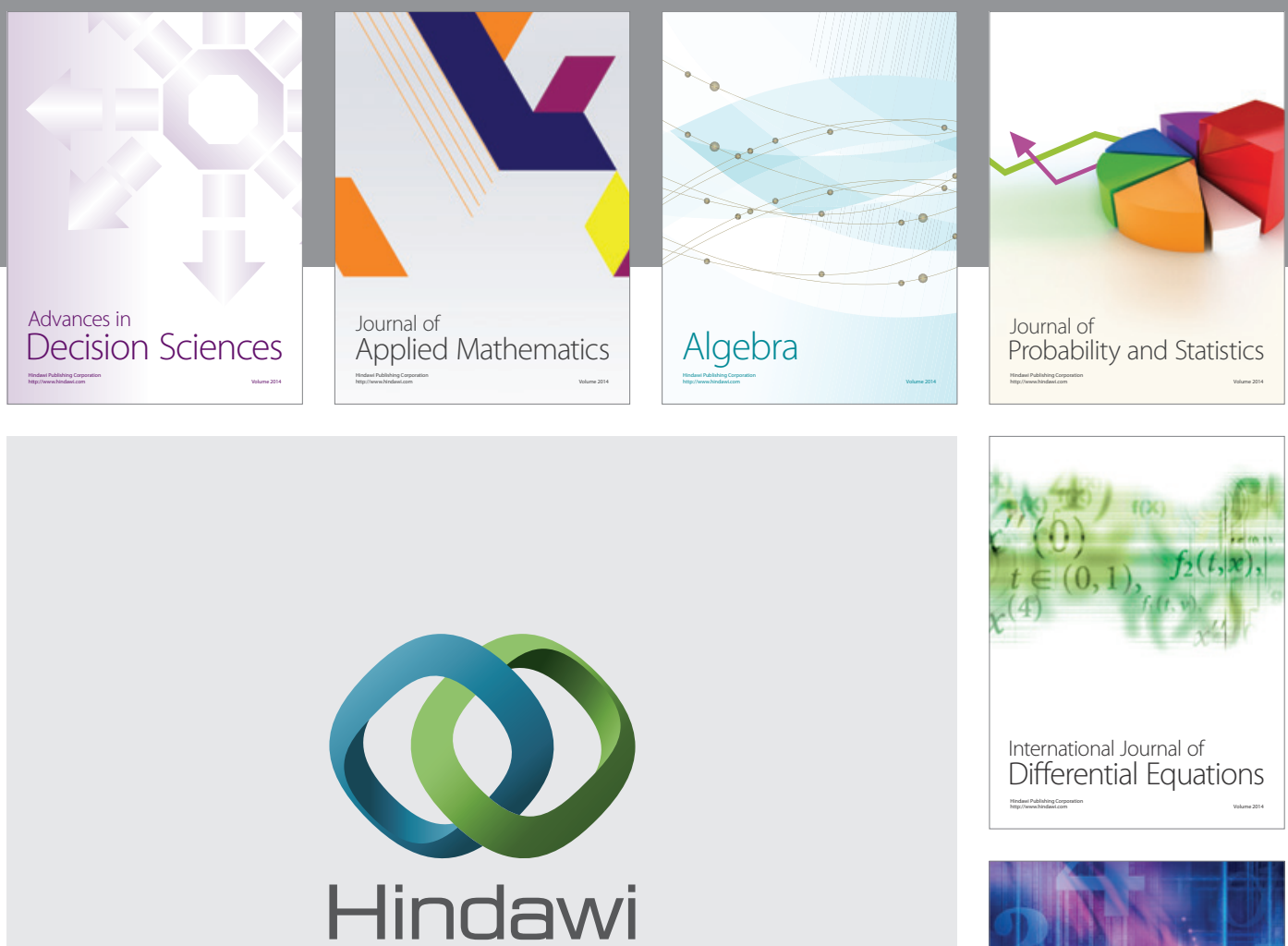

Submit your manuscripts at http://www.hindawi.com
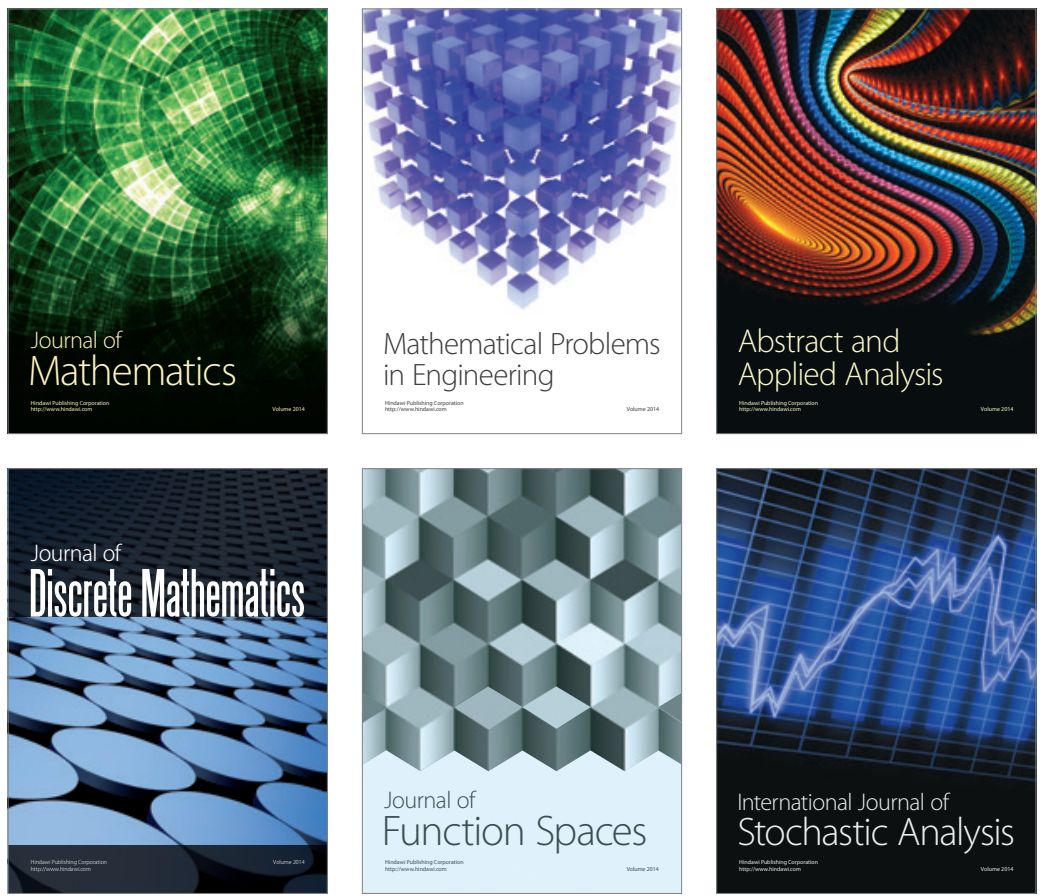

Journal of

Function Spaces

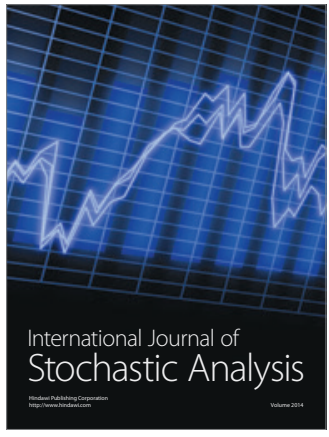

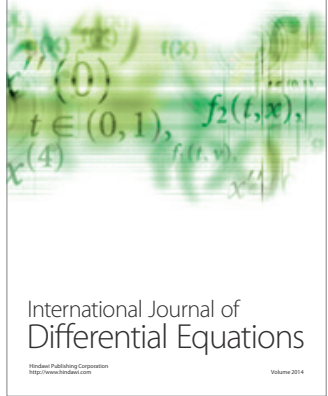
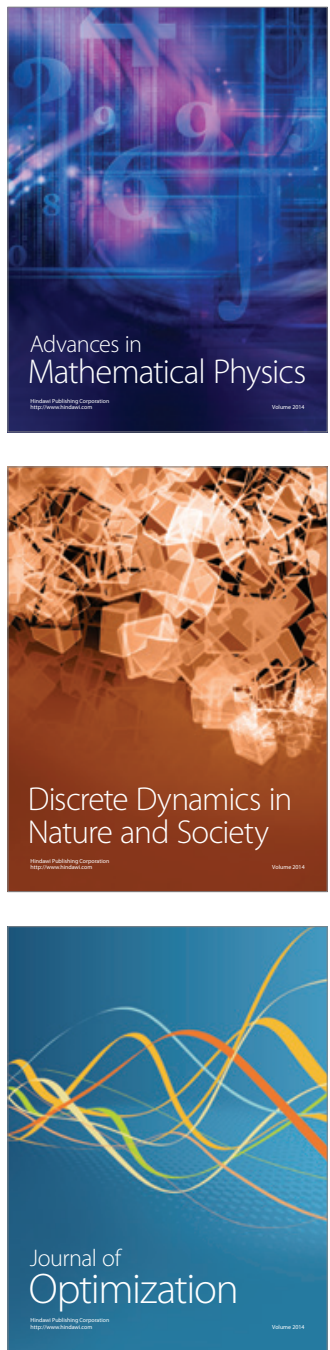